\title{
Hot Electron Capture Dissociation Distinguishes Leucine from Isoleucine in a Novel Hemoglobin Variant, Hb Askew, B54(D5) Val $\rightarrow$ Ile
}

\author{
Jonathan P. Williams, ${ }^{a}$ Andrew J. Creese, ${ }^{b}$ David R. Roper, ${ }^{c}$ \\ Brian N. Green, ${ }^{d}$ and Helen J. Cooper ${ }^{b}$ \\ a Department of Chemistry, University of Warwick, Coventry, United Kingdom \\ ${ }^{\mathrm{b}}$ School of Biosciences, College of Life and Environmental Sciences, University of Birmingham, Edgbaston, \\ United Kingdom \\ ${ }^{\mathrm{c}}$ Department of Hematology, Imperial College Healthcare NHS Trust, Hammersmith Hospital, London, \\ United Kingdom \\ d Waters MS Technologies Centre, Micromass UK Ltd., Cheshire, United Kingdom
}

\begin{abstract}
Population migration has led to the global dispersion of human hemoglobinopathies and has precipitated a need for their identification. An effective mass spectrometry-based procedure involves analysis of the intact $\alpha$ - and $\beta$-globin chains to determine their mass, followed by location of the variant amino acid residue by direct analysis of the enzymatically digested chains and low-energy collision induced dissociation of the variant peptide. Using this procedure, a variant was identified as either $\beta 54 \mathrm{Val} \rightarrow$ Leu or $\beta 54 \mathrm{Val} \rightarrow \mathrm{Ile}$, since the amino acids leucine and isoleucine cannot be distinguished using low-energy collisions. Here, we describe how hot electron capture dissociation on a Fourier transform-ion cyclotron resonance mass spectrometer was used to distinguish isoleucine from leucine and identify the mutation as $\beta 54(\mathrm{D} 5) \mathrm{Val} \rightarrow$ Ile. This is a novel variant, and we have named it $\mathrm{Hb}$ Askew. (J Am Soc Mass Spectrom 2009, 20, 1707-1713) (c) 2009 American Society for Mass Spectrometry
\end{abstract}

$\mathrm{H}$ emoglobin $(\mathrm{Hb})$ exists in the blood cells of vertebrates as a noncovalently assembled tetramer of $\alpha$ - and $\beta$-chains $\left(\alpha_{2} \beta_{2}\right)$, in which each chain is associated with a heme group. Its primary function is to supply oxygen to the organs of the body. Abnormalities of human hemoglobin $(\mathrm{Hb})$ are the most common autosomal recessive inherited disorders in man. These disorders can be both quantitative, due to impaired synthesis of the globin chains (thalassemia syndromes), or qualitative (structural variants). Many hemoglobinopathies do not cause clinical problems, but some are responsible for morbidity and mortality. $\mathrm{Hb}$ variants initially tended to be located with highfrequency in tropical and subtropical regions such as Africa, the Caribbean, the Mediterranean, Asia, and the Far East. The large degree of population migration has led to the worldwide spread of $\mathrm{Hb}$ disorders.

$\mathrm{Hb}$ variants are often detected as part of a routine glycohemoglobin screen during diabetic monitoring or antenatal and neonatal screening healthcare programs. Hemoglobinopathy diagnosis in clinical laboratories is

Address reprint requests to Dr. J. P. Williams, Department of Chemistry, University of Warwick, Coventry, CV4 7AL, UK. E-mail: J.P.Williams@ warwick.ac.uk or to Dr. H. J. Cooper, School of Biosciences, University of Birmingham, Edgbaston, Birmingham, B15 2TT, UK. E-mail: H.J.Cooper@ bham.ac.uk routinely accomplished using isoelectric focusing (IEF) or cation exchange high-performance liquid chromatography (ce-HPLC) procedures. These methods presumptively identify variants but cannot positively identify any variant. Precise variant identification requires protein sequencing or DNA analysis. The majority of $\mathrm{Hb}$ variants listed in the Globin Gene Server [1] database result from a single point mutation in either the $\alpha$ - or $\beta$ globin gene that leads to the production of a single amino acid substitution in either the $\alpha$ - or $\beta$-chain of the $\mathrm{Hb}$ protein. Although many of these variants may be innocuous, once detected in a first-line hospital screen, it is sensible to precisely identify the mutation.

A procedure for identifying $\mathrm{Hb}$ variants by electrospray ionization mass spectrometry (ESI-MS) has been described previously $[2,3]$. There are three steps in this procedure. The first step involves analyzing blood diluted in a denaturing solvent to determine the molecular weight of the variant chain and assign the variant to the $\alpha$ - or $\beta$-chain. In the second step, diluted blood is digested with trypsin and the resulting mixture of peptides analyzed directly by ESI-MS. The third step involves sequencing the variant tryptic peptide by tandem mass spectrometry if required. No chromatographic separation of the tryptic peptides before or during analysis is required. The accurate average mass 
measurement involved in step one allows for the detection of variants in heterozygotes that differ by as little as $1 \mathrm{Da}$ from normal, providing the variant is present at $>10 \%$ relative abundance $[3,4]$. Using this procedure and applying the $\alpha$-chain mass for internal calibration, the normal $\beta$-chain mass can be determined with a precision of $\leq 0.03$ Da standard deviation [4]. Important minor $\mathrm{Hb}$ fractions, such as $\mathrm{HbA}_{1 \mathrm{C}}$ (assessment of long-term glycemic control in diabetics) [5-7] and $\mathrm{HbA}_{2}$ (biomarker for $\beta^{0}$-thalassemia trait) [8], are also detected and quantified using these procedures following calibration with standards.

The above procedure involves sequencing peptides via low-energy collision-induced dissociation (CID) in a triple quadrupole instrument, and $>95 \%$ of the variants encountered in practice can be identified. However, there are a number of variants that involve amino acid exchanges governed by single mutations in the nucleotide codon to either leucine (Leu) or isoleucine (Ile), which cannot be differentiated using this procedure. Leu and Ile are isomeric species. Therefore, low-energy CID of variant peptide precursor ions, which contain either Leu or Ile, generates sequence ions [9] of the same $\mathrm{m} / \mathrm{z}$ and renders unequivocal assignment of these species impossible.

Here, we describe a mass spectrometric approach, which allows distinction between Leu and Ile when characterizing human hemoglobinopathies. This approach utilizes hot electron capture dissociation (HECD) [10] on a Fourier transform ion cyclotron resonance (FT-ICR) mass spectrometer. In conventional (low-energy) ECD $[11,12]$, peptide backbone cleavage occurs at the N-C $\alpha$ bond to produce $c^{\prime}$ and $z \bullet$ ions. These fragments also result from HECD but are accompanied by extensive secondary fragmentation due to the excess energy. Secondary fragmentation of amino acid side chains of $z \bullet$ ions result in the formation of $w$ ions. The loss of - $\mathrm{CH}\left(\mathrm{CH}_{3}\right)_{2}(-43 \mathrm{Da})$ or $\bullet \mathrm{CH}_{2} \mathrm{CH}_{3}(-29 \mathrm{Da})$ from $z \bullet$ ions containing $\mathrm{N}$-terminal Leu or Ile, respectively, enables the two amino acids to be distinguished, see Scheme 1. This method has been demonstrated on synthetic peptides [10] and the bovine milk protein PP3 [13]. In the present example, a blood sample was found to contain three variants, one of which involved a mutation to either Leu or Ile. Here we show how HECD was used to resolve the ambiguity and identify a novel $\beta$-chain variant. To confirm the assignment, HECD of Leu- and Ile-containing synthetic analogues of the tryptic peptide containing the variant was also performed. This work demonstrates the significant potential role of HECD in the characterization of these types of $\mathrm{Hb}$ mutation, and also constitutes the first true clinical application of the HECD technique.

\section{Methods}

\section{Cation-Exchange HPLC}

Cation-exchange HPLC with UV detection was performed on a VARIANT HPLC system using the Variant $\beta$-thalassemia Short program (Bio-Rad Laboratories Ltd., Hemel Hempstead, Hertfordshire, UK).

\section{Sample Preparation}

The procedures for identifying variants in blood samples by mass spectrometry have been previously described in detail elsewhere [2,3]. Briefly, $10 \mu \mathrm{L}$ of the blood sample (in ethylene diamine tetra-acetic acid (EDTA) anti-coagulant) was diluted 50-fold with $490 \mu \mathrm{L}$ of water to give a stock solution. Then, $20 \mu \mathrm{L}$ of the stock solution was diluted a further 10 -fold with $180 \mu \mathrm{L}$ of $5: 4$ acetonitrile:water containing $0.2 \%$ formic acid (Solution A). Tryptic digests were prepared as follows: first, $100 \mu \mathrm{L}$ of the stock solution was denatured by mixing with $20 \mu \mathrm{L}$ of $50 \%$ aqueous acetonitrile containing $0.5 \%$ formic acid. Then, $6 \mu \mathrm{L}$ of $1 \mathrm{M}$ ammonium bicarbonate solution was added, followed by $5 \mu \mathrm{L}$ of a

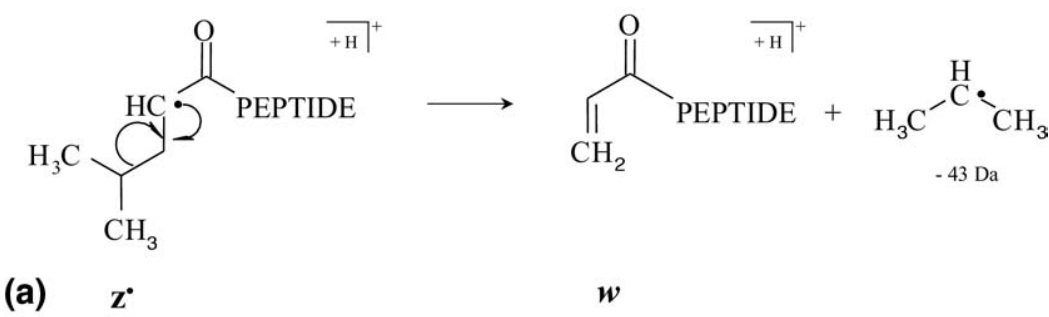

(a)
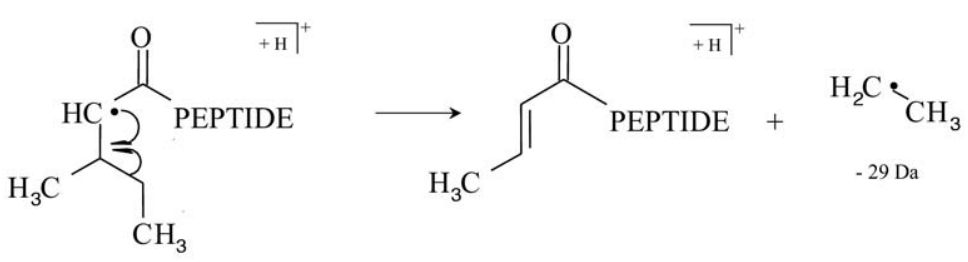

(b)

$\mathbf{z}^{*}$

$\boldsymbol{w}$

Scheme 1. Secondary fragmentation of a $z \bullet$ ion to produce the $w$ ion from a peptide containing (a)

a leucine residue and (b) an isoleucine residue. 
$5 \mathrm{mg} / \mathrm{mL}$ solution of trypsin (T1426, Sigma-Aldrich Corp., St. Louis, MO, USA). The resulting solution was then incubated at $37^{\circ} \mathrm{C}$ for $30 \mathrm{~min}$, after which aliquots were diluted 10 -fold with Solution A.

The peptides FFESFGDLSTPDAIMGNPK and FFESFGDLSTPDALMGNPK were synthesized by AltaBioscience, University of Birmingham, UK, and used without further purification. The peptides were diluted to 2 $\mathrm{pmol} / \mu \mathrm{L}$ in methanol (Fisher Scientific, Leicestershire, UK):water (J. T. Baker, Deventer, The Netherlands) (75:25) with $1 \%$ formic acid (Fisher Scientific).

\section{Mass Spectrometry}

ESI-MS was performed on two instruments. Initial experiments were undertaken on a triple quadrupole mass spectrometer (Quattro Ultima; Waters MS Technologies, Manchester, UK) equipped with the standard Z-spray electrospray ion source and operated at a source and desolvation temperature of $110^{\circ} \mathrm{C}$. Sample solutions were introduced into the source region of the instrument at a rate of $5 \mu \mathrm{L} / \mathrm{min}$. The instrument was operated in positive mode of ionization, with a capillary voltage of $3 \mathrm{kV}$, a cone voltage of $80 \mathrm{~V}$, and hexapole 1 ion guide set to $60 \mathrm{~V}$. Tandem mass spectrometry (MS/MS) was carried out using argon collision gas at a pressure of $2.5 \times 10^{-3}$ mbar within the RF-only hexapole collision cell. Fragmentation of the precursor tryptic peptide ions was achieved with a collision energy of $30 \mathrm{eV}$.

FT-ICR analysis was performed on a Thermo Finnigan LTQ FT mass spectrometer (Thermo Fisher Scientific, Bremen, Germany). Samples were injected by use of an Advion Biosciences Triversa electrospray source (Advion Biosciences, Ithaca, NY, USA) at a flow rate of $\sim 200 \mathrm{~nL} / \mathrm{min}$. For all experiments, scans were acquired in the ICR cell with a resolution of 100,000 at $\mathrm{m} / \mathrm{z} 400$. ECD and HECD of tryptic peptide: precursor ions were isolated in the linear ion trap and transferred to the ICR cell for $(\mathrm{H}) \mathrm{ECD}$. AGC target was $1 \times 10^{6}$. Isolation width was $6 \mathrm{Th}$. The electrons for $(\mathrm{H}) \mathrm{ECD}$ were produced by an indirectly heated barium-tungsten cylindrical dispenser cathode $(5.1 \mathrm{~mm}$ diameter, $154 \mathrm{~mm}$ from the cell, $1 \mathrm{~mm}$ off axis) (Heat-Wave Labs, Watsonville, CA, USA). The current across the electrode was $\sim 1.1 \mathrm{~A}$. Ions were irradiated for $70 \mathrm{~ms}$ at $5 \%$ energy (ECD) (corresponding to ECD cathode potential -4.35 $\mathrm{V})$ or $15 \%$ energy (HECD) (corresponding to $\mathrm{ECD}$ cathode potential -14.35 V). Each (H)ECD scan comprised four co-added microscans. Mass spectra shown comprise fifty averaged scans. HECD of synthetic peptides: instrumental parameters were as above, except that the current across the electrode was $\sim 1.0 \mathrm{~A}$. Ions were irradiated for $70 \mathrm{~ms}$ at $17.5 \%$ energy (corresponding to ECD cathode potential $-16.18 \mathrm{~V}$ ). Each HECD scan comprised 20 co-added microscans. Mass spectra shown comprise one scan.

\section{Results}

A blood sample was submitted for investigation by mass spectrometry because abnormalities had been detected during a routine antenatal screen in a hospital hematology laboratory using ce-HPLC. The ce-HPLC chromatogram (Figure 1) showed two abnormal peaks, which eluted after the time for normal adult $\mathrm{Hb}$ (A0, $2.44 \mathrm{~min})$. The elution time of the earlier peak $(4.50 \mathrm{~min})$ corresponds to that of the clinically significant Sickle variant, but the peak itself is atypical in that there appear to be shoulders on both its leading and trailing edges. A sickle cell solubility test was positive. Apart from the unusual ce-HPLC chromatogram, no other hematological abnormalities were observed. To clarify these anomalies, the sample was submitted for analysis by ESI-MS.

Initially, mass spectrometric analysis was performed on a triple quadrupole instrument. Analysis of the intact $\mathrm{Hb}$ chains revealed the presence of one $\alpha$-chain variant and, surprisingly, two $\beta$-chain variants (Figure 2 ). The $\alpha$-chain variant was $14 \mathrm{Da}$ heavier than normal and its proportion of total $\alpha$-chains was $28.3 \%$. It was identified from a 30-min tryptic digest as $\mathrm{Hb}$ Stanleyville II $(\alpha 78\{$ EF7\}Asn $\rightarrow$ Lys). The lighter $\beta$-chain variant (normal $\beta-30 \mathrm{Da}$ and $42.2 \%$ of total $\beta$-chains) was confirmed from the same digest as $\mathrm{Hb}$ Sickle $(\beta 6\{\mathrm{~A} 3\} \mathrm{Glu} \rightarrow \mathrm{Val})$. The unexpected third variant was $14 \mathrm{Da}$ heavier than the normal $\beta$-chain and its mutant amino acid was shown from the spectrum of the tryptic digest to occur in the $\beta T 5$ peptide (Figure 3). There are nine potential mutations in this peptide that could give a $14 \mathrm{Da}$ mass increase by a single base change in the nucleotide codon. To distinguish these possibilities, the normal and variant $\beta \mathrm{T} 5^{2+}$ ions were sequenced by tandem mass spectrometry using low-energy CID (Figure 4). The $14 \mathrm{Da}$ mass increase at $\mathrm{y}^{\prime \prime}{ }_{6}$ between the spectra from (a) the normal peptide and (b) the variant peptide placed the mutation at $\beta 54$, but did not allow $\beta 54 \mathrm{Val} \rightarrow$ Leu to be distinguished from $\beta 54 \mathrm{Val} \rightarrow$ Ile.

To precisely characterize the mutation and resolve the ambiguity between these two isomeric amino acids, the variant $\beta \mathrm{T}^{2+}$ ion was subjected to hot ECD. The ECD mass spectrum obtained under standard operating

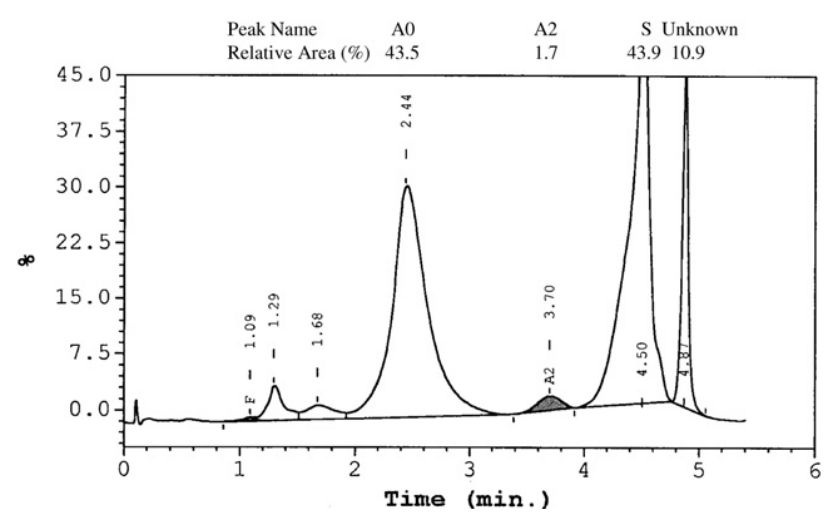

Figure 1. Cation-exchange HPLC trace of the abnormal blood sample. Peak names are those assigned by the ce-HPLC system. A0: normal hemoglobin. 


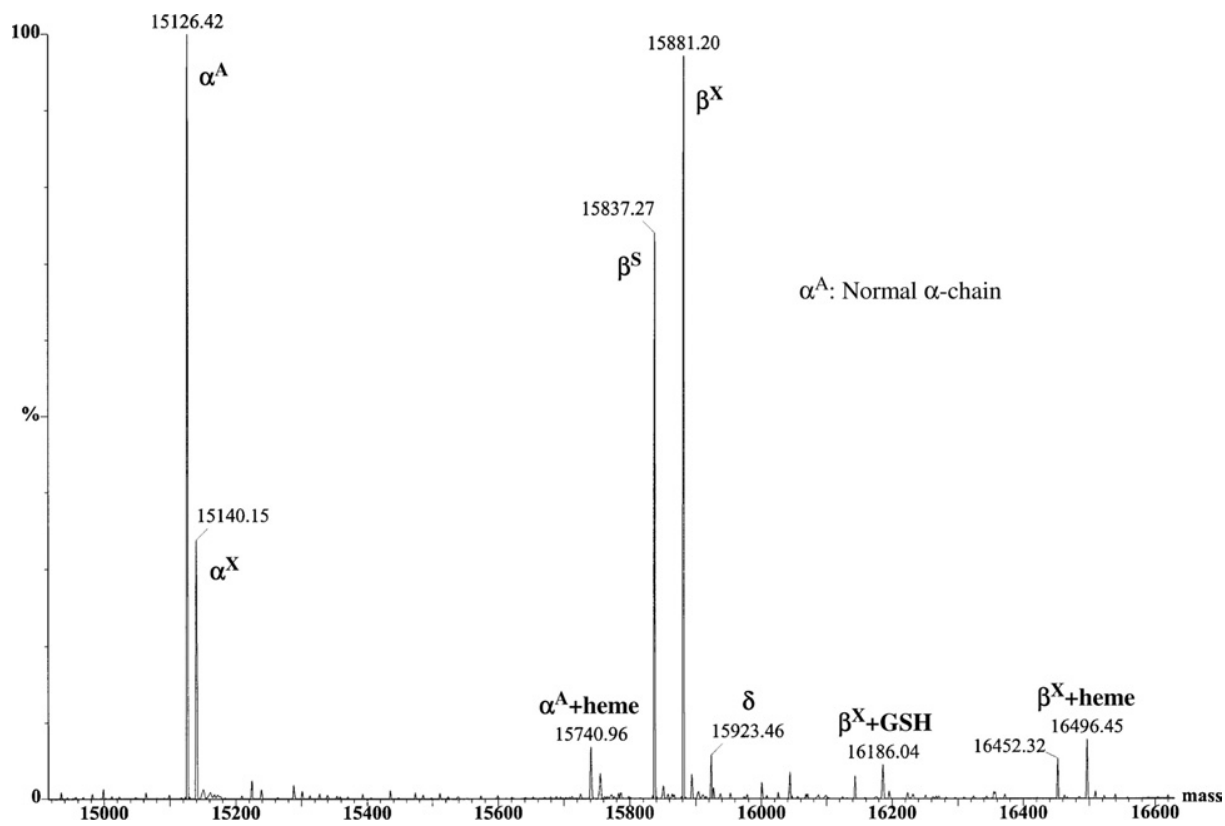

Figure 2. Maximum entropy deconvoluted ESI mass spectrum of the 500-fold diluted and denatured abnormal blood sample obtained by the triple quadrupole instrument. It shows the presence of a 14 Da heavier than normal $\alpha$-chain variant $\left(\alpha^{\mathrm{X}}\right)$, and two $\beta$-chain variants, one $30 \mathrm{Da}$ lighter $\left(\beta^{\mathrm{S}}\right)$ and the other $14 \mathrm{Da}$ heavier than normal $\left(\beta^{\mathrm{X}}\right)$. Masses are experimental. The sequence masses of the normal $\alpha$ - and $\beta$-chains are $15,126.38$ and $15,867.24 \mathrm{Da}$, respectively. GSH: glutathione.

conditions (ECD energy $=5 \%$, corresponding to $\mathrm{ECD}$ cathode potential $=-4.35 \mathrm{~V})$ (data not shown) did not allow differentiation between the isomeric amino acids. Interestingly, $z_{6}$ ions were not observed in that mass spectrum. At higher electron energies (ECD energy = $15 \%$, corresponding to ECD cathode potential $=-14.35$ V) (Figure 5), additional fragmentation was detected. A peak corresponding to the $z_{6}{ }^{\prime}$ ion $\left(m / z_{\text {meas }} 644.3446\right.$, $\left.m / z_{\text {calc }} 644.3436\right)$ was observed, as was a peak corresponding to $\left(z_{6} \bullet-29\right)$, (see insets). (Note that $z^{\prime}$ ions are the result of hydrogen abstraction by radical $z \bullet$ ions. In the present case, apparently the $z_{6} \bullet$ ions either fragment producing $w$ ions, or hydrogen abstraction occurs resulting in $z_{6}{ }^{\prime}$ ions). No peak corresponding to $\left(z_{6} \bullet-43\right)$ was observed at $m / z 600$. As described above, the $z_{6} \bullet-29$ ion constitutes a secondary $w$ ion originat-

(a)

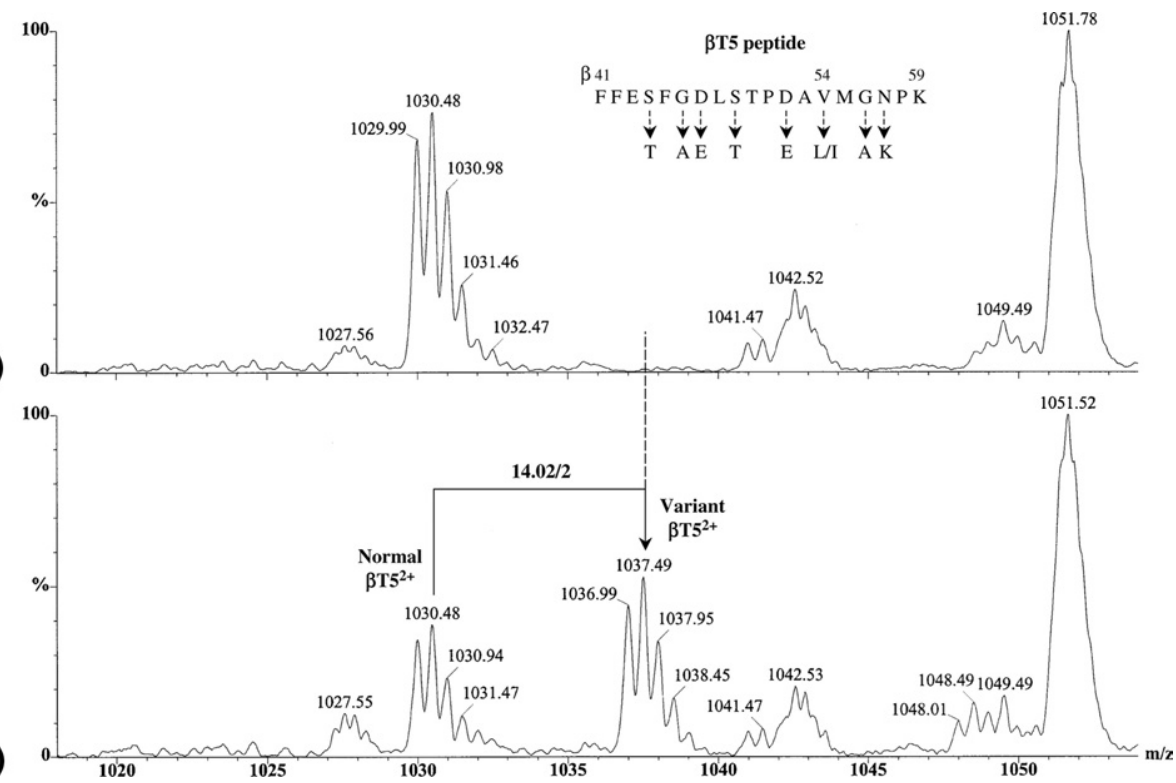

Figure 3. Diagnostic region of mass spectra obtained by the triple quadrupole instrument from 30-min tryptic digests of (a) a normal control sample and (b) the abnormal sample showing that the mutation in the heavier than normal $\beta$-chain variant occurs in the $\beta \mathrm{T} 5$ peptide. 


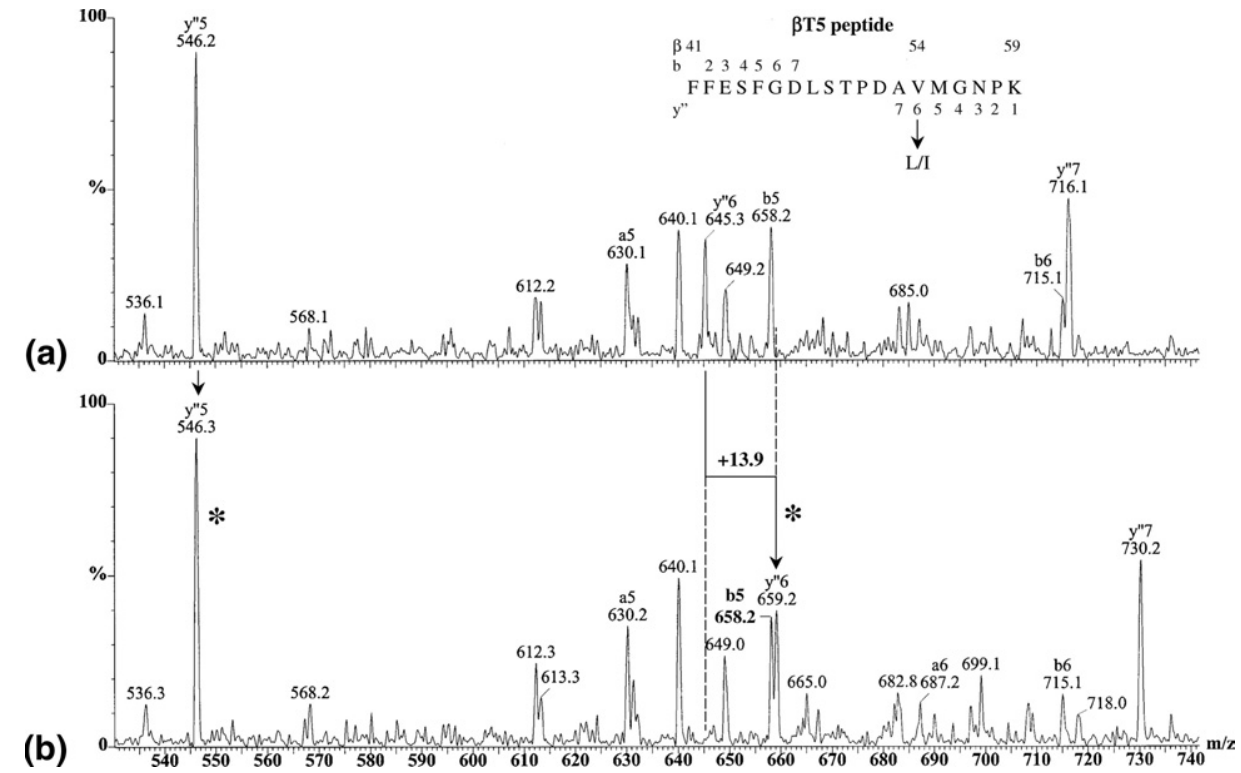

Figure 4. Diagnostic region of product ion spectra obtained using the triple quadrupole from (a) the normal $\beta \mathrm{T}^{2+}$ peptide ion and (b) the variant $\beta \mathrm{T} 5^{2+}$ peptide ion. The 14 Da mass-increase between (a) and (b) at $\mathrm{y}_{6}{ }_{6}$ identifies the mutation as either $\beta 54 \mathrm{Val} \rightarrow$ Leu or $\beta 54 \mathrm{Val} \rightarrow$ Ile.

ing from an isoleucine residue (Scheme 1). To confirm our assignment, we performed HECD on the synthetic peptides FFESFGDLSTPDAIMGNPK and FFESFGDLSTPDALMGNPK (Figure 6). As for the $\beta \mathrm{T} 5^{2+}$ ions, HECD of $[\mathrm{M}+2 \mathrm{H}]^{2+}$ ions of FFESFGDLSTPDAIMGNPK (Figure 6a) resulted in a peak corresponding to $\left(z_{6} \bullet-29\right)$ ions. No peak corresponding to $\left(z_{6} \bullet-43\right)$ ions was observed. HECD of $[\mathrm{M}+2 \mathrm{H}]^{2+}$ ions of FFESFGDLSTPDALMGNPK (Figure $6 b$ ) resulted in a peak corresponding to $\left(z_{6} \bullet-43\right)$ ions. No peak corresponding to $\left(z_{6} \bullet-29\right)$ ions was observed. The mutation is thus identified as $\beta 54(\mathrm{D} 5) \mathrm{Val} \rightarrow$ Ile. This mutation has not been described previously [1], and we have named it $\mathrm{Hb}$ Askew.

\section{Discussion}

As a stand alone technique ce-HPLC may presumptively, but never positively, identify any variant. Mass

\section{FFESEGDLSTIPDAIMGANPK}

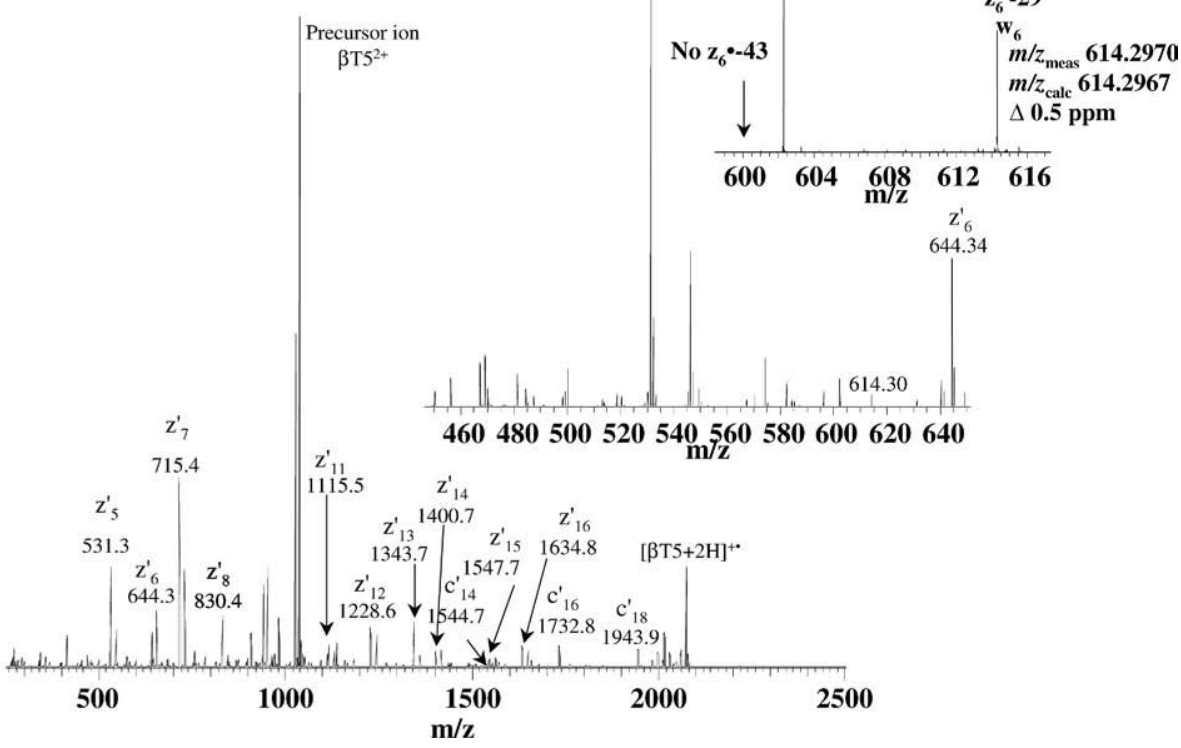

Figure 5. HECD mass spectra of the variant $\beta T 5^{2+}$ peptide ion obtained using the FT-ICR instrument. The insets show the detection of the $w_{6}\left(z_{6}-29 \mathrm{Da}\right)$ ion which identifies the mutation as $\beta 54(\mathrm{D} 5) \mathrm{Val} \rightarrow \mathrm{Ile}$. 
(a)
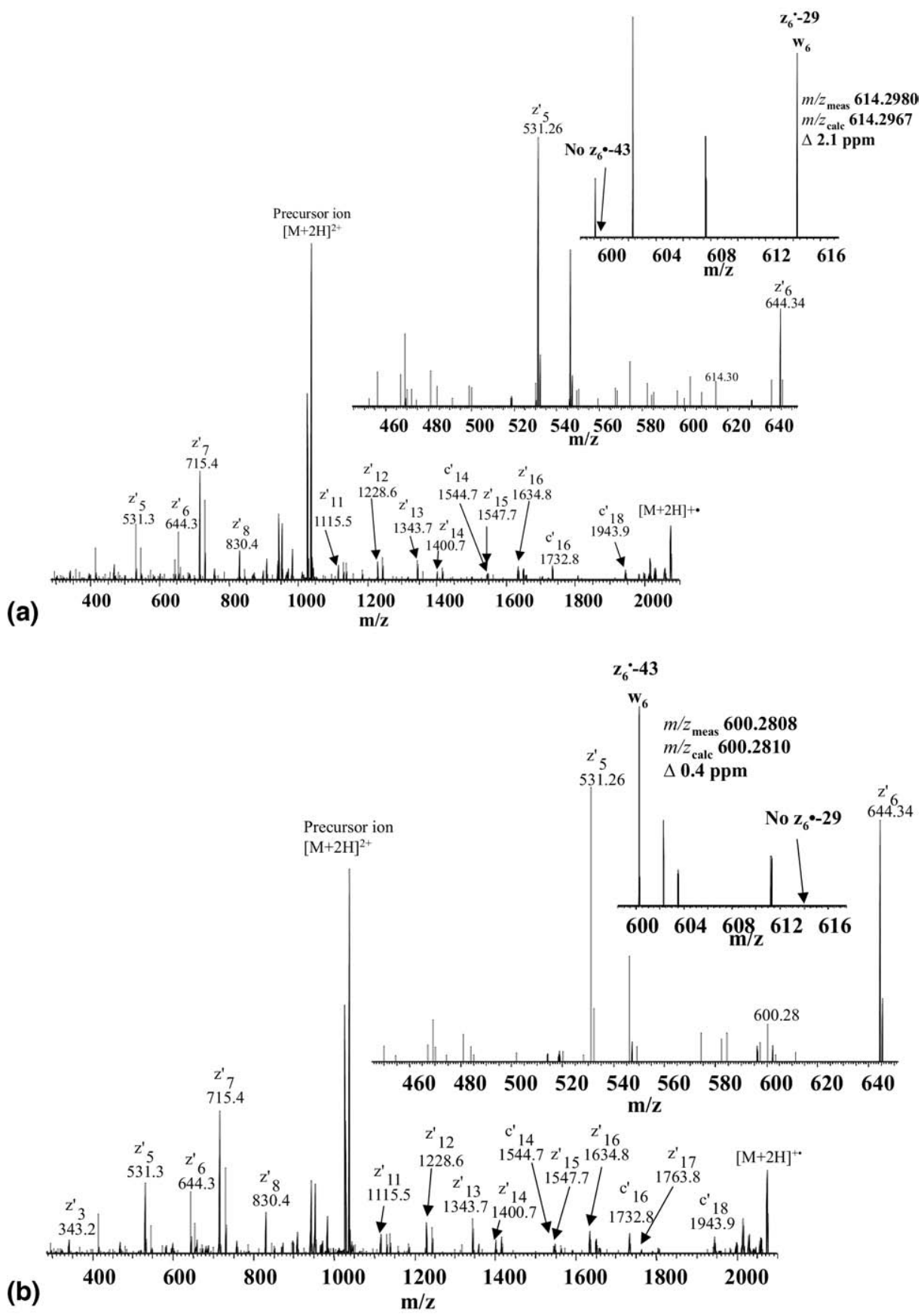

Figure 6. HECD mass spectra of the $[\mathrm{M}+2 \mathrm{H}]^{2+}$ ions of the synthetic peptides (a) FFESFGDLSTPDAIMGNPK and (b) FFESFGDLSTPDALMGNPK. The insets show the detection of the $w_{6}$ ions.

spectrometry has been shown to be a powerful complementary technique, which can detect and identify phenotypically silent mutations as well as those that are easily detected by ce-HPLC [8]. In this study, the mutation $\beta 54$ (D5)Val $\rightarrow$ Ile is silent by ce-HPLC and was only discovered because it occurred together with other detectable mutations in the same patient, so prompting further investigation. The aim of the UK antenatal screening program is to detect Sickle $\mathrm{Hb}, \beta$-thalassemia trait, or one of the clinically significant variants, such as $\mathrm{Hb} \mathrm{C}$, D-Punjab, E, O-Arab, and Lepore, which are known to interact with Sickle to cause sickling. The initial 3 min analysis of the intact $\mathrm{Hb}$ chains supported the likely presence of the Sickle variant and ruled out the presence of any of the above known interacting variants. The results also demonstrate the utility of HECD for distinguishing between Leu and Ile residues in human hemoglobinopathies obviating the need for DNA analysis. The variant was initially identified as $\beta 54 \mathrm{Val} \rightarrow$ Leu or Ile by triple quadrupole mass spectrometry. Low-energy CID on the triple quadrupole and FT-ICR instruments, and conventional ECD could 
not resolve the ambiguity as backbone sequence fragments from the isomers have identical masses. HECD results in secondary fragmentation of the amino acid side-chains allowing Leu and Ile to be distinguished. In the present case, we identified the valine to isoleucine mutation at position 54 in the $\beta$-chain following detection of the $w_{6}\left(z_{6} \bullet-29\right)$ ion from the peptide FFESFGDLSTPDAIMGNPK, and the absence of a $w_{6}\left(z_{6} \bullet-43\right)$ ion that would have implied the tryptic peptide FFESFGDLSTPDALMGNPK. The assignment was confirmed by performing HECD on the synthetic analogues of these peptides. The codon for the $\beta 54 \mathrm{Val}$ is GTT. The codons for Ile are ATT, ATC, and ATA, and those for Leu are TTA, TTG, CTT, CTC, CTA, and CTG. Thus we conclude that the Askew variant is the result of a single base change in the nucleotide codon, GTT $\rightarrow$ ATT.

\section{Acknowledgments}

JPW thanks the Warwick University Research Development Fund for a grant and Professor Peter J. Sadler for helpful discussions. HJC gratefully acknowledges the Wellcome Trust (074131) for funding.

\section{References}

1. Hardison, R. C.; Chui, D. H. K.; Giardine, B.; Riemer, C.; Patrinos, G. P.; Anagnou, N.; Millar, W.; Wajcman, H. HbVar: A relational database of human hemoglobin variants and thalassemia mutations at the globin gene server. Human Mutat. 2002, 19, 225-233. (http://globin.cse. psu.edu).
2. Wild, B. J.; Green, B. N.; Cooper, E. K.; Lalloz, M. R. A.; Erten, S. Stephens, A. D.; Layton, D. M. Rapid identification of hemoglobin variants by electrospray ionization mass spectrometry. Blood Cells $\mathrm{Mol}$. Dis. 2001, 27, 691-704.

3. Rai, D. K.; Griffiths, W. J.; Landin, B.; Wild, B. J.; Alvelius, G.; Green, B. N. Accurate mass measurement by electrospray ionization quadrupole mass spectrometry: Detection of variants differing by $<6$ Da from normal in human hemoglobin heterozygotes. Anal. Chem. 2003, 75, 1978-1982.

4. Bateman, R. H.; Green, B. N.; Morris, M. Electrospray ionization mass spectrometric analysis of the globin chains in hemoglobin heterozygotes can detect the variants $\mathrm{HbC}, \mathrm{D}$, and E. Clin. Chem. 2008, 54, 1256-1257.

5. Roberts, N. B.; Green, B. N.; Morris M. Potential of electrospray mass spectrometry for quantifying glycohemoglobin. Clin. Chem. 1997, 43, 771-778.

6. Roberts, N. B.; Amara, A. B.; Morris, M.; Green, B. N. Long-term evaluation of electrospray ionization mass spectrometric analysis of glycated hemoglobin. Clin. Chem. 2001, 47, 316-321.

7. Williams, J. P., Jackson, H., Green, B. N. Hb Belleville [ $\beta 10(\mathrm{~A} 7) \mathrm{Ala} \rightarrow \mathrm{Thr}$ ] affects the determination of $\mathrm{Hb} \mathrm{A}_{1 \mathrm{C}}$ by routine cation exchange high performance liquid chromatography. Hemoglobin 2009, 33, 45-50.

8. Williams, J. P.; Scrivens, J. H.; Green, B. N.; Farrar, L. M.; Sutcliffe, M. $\mathrm{Hb}$ Leeds [ $\beta 56(\mathrm{D} 7) \mathrm{Gly} \rightarrow \mathrm{Cys}$ ]: A new hemoglobin that aggravates anemia in a child with $\beta^{0}$-thalassemia trait. Hemoglobin 2007, 31, 367-373.

9. Roepstorff, P.; Fohlman, J. Proposal for a common nomenclature for sequence ions in mass spectra of peptides. Biol. Mass Spectrom. 1984, 11, 601.

10. Kjeldsen, F.; Haselmann, K. F.; Budnik, B. A.; Jensen, F.; Zubarev, R. A. Dissociative capture of hot (3-13 eV electrons by polypeptide polycations: An efficient process accompanied by secondary fragmentation. Chem. Phys. Lett. 2002, 356, 201-206.

11. Cooper, H. J.; Hakansson, K.; Marshall, A. G. The role of electron capture dissociation in biomolecular analysis. Mass Spectrom. Rev. 2005, 24, 201-222.

12. Zubarev, R. A.; Kelleher, N. L.; McLafferty, F. W. ECD of multiply charged protein cations. A nonergodic process. J. Am. Chem. Soc. 1998 120, 3265-3266.

13. Kjeldsen, F.; Haselmann, K. F.; Sorensen, E. S.; Zubarev, R. A. Distinguishing of Ile/Leu amino acid residues in the PP3 protein by (hot) electron capture dissociation in Fourier transform ion cyclotron resonance mass spectrometry. Anal. Chem. 2003, 75, 1267-1274. 